\title{
Unilateral global interval bifurcation theorem for periodic $p$-Laplacian and its applications
}

Wenguo Shen ${ }^{*}$ and Tao He

\begin{abstract}
In this paper, we establish a unilateral global bifurcation result from interval for a class of periodic $p$-Laplacian problems with non-differentiable nonlinearity. By applying the above result, we shall prove the existence of the principal half-eigenvalues for the half-quasilinear periodic $p$-Laplacian problem

$$
\begin{aligned}
& -\left(\varphi_{p}\left(x^{\prime}\right)\right)^{\prime}+q(t) \varphi_{p}(x)=\lambda a(t) \varphi_{p}(x)+\alpha(t) \varphi_{p}\left(x^{+}\right)+\beta(t) \varphi_{p}\left(x^{-}\right), \quad 0<t<T, \\
& x(0)=x(T), \quad x^{\prime}(0)=x^{\prime}(T),
\end{aligned}
$$
\end{abstract}

where $\varphi_{p}(s)=|s|^{p-2} s, \lambda$ is a positive parameter and $x^{+}=\max \{x, 0\}, x^{-}=-\min \{x, 0\}$, $a(t) \in C([0, T],(0, \infty)), \alpha(t), \beta(t) \in C[0, T]$. Moreover, we also investigate the existence of one-sign solutions for a class of half-quasilinear periodic $p$-Laplacian problem

$$
\begin{aligned}
& -\left(\varphi_{p}\left(x^{\prime}\right)\right)^{\prime}+q(t) \varphi_{p}(x)=\alpha(t) \varphi_{p}\left(x^{+}\right)+\beta(t) \varphi_{p}\left(x^{-}\right)+r a(t) f(x), \quad 0<t<T, \\
& x(0)=x(T), \quad x^{\prime}(0)=x^{\prime}(T),
\end{aligned}
$$

where $f \in C(\mathbb{R}, \mathbb{R})$, sf $(s)>0$ for $s \neq 0$, and $f_{0} \in(0, \infty]$ and $f_{\infty} \in(0, \infty)$ or $f_{0} \in[0, \infty]$ and $f_{\infty}=\infty$, where $f_{0}=\lim _{|s| \rightarrow 0} f(s) / \varphi_{p}(s), f_{\infty}=\lim _{|s| \rightarrow+\infty} f(s) / \varphi_{p}(s)$.

MSC: 34B15;34C10;34C23

Keywords: unilateral interval bifurcation; half-quasilinear problems; one-sign solutions; periodic $p$-Laplacian problems

\section{Introduction}

Let $E$ be a real Banach space with the norm $\|\cdot\|$. Consider the operator equation

$$
u=\lambda B u+H(\lambda, u)
$$

where $B$ is a compact linear operator and $H: \mathbb{R} \times E \rightarrow E$ is compact with $H=o(\|u\|)$ at $u=0$ uniformly on bounded $\lambda$ intervals. Krasnoselskii [1] has shown that all characteristic values of $B$ which are of odd multiplicity are bifurcation points. Rabinowitz [2] has extended this result by showing that bifurcation has global consequences. Furthermore, if

\section{Springer}


the characteristic value $\mu$ of $B$ has multiplicity 1 and

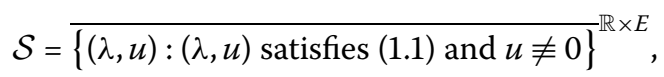

Dancer [3] has shown that there are two distinct unbounded continua $\mathscr{C}_{\mu}^{+}$and $\mathscr{C}_{\mu}^{-}$, consisting of the bifurcation branch $\mathscr{C}_{\mu}$ of $\mathcal{S}$ emanating from $(\mu, 0)$, which satisfy either $\mathscr{C}_{\mu}^{+}$ and $\mathscr{C}_{\mu}^{-}$are both unbounded or $\mathscr{C}_{\mu}^{+} \cap \mathscr{C}_{\mu}^{-} \neq\{(\mu, 0)\}$.

On the other hand, in the past few decades, the periodic solutions of the well-known Camassa-Holm equation have been studied by some specialists, see [4-10]. By the fixed point theory in the cones, some authors [11-17] have also considered periodic problems. Recently, Ma et al. $[18,19]$ studied the existence of positive solutions for the periodic problems by applying bifurcation techniques. Especially, in 2012, Dai et al. [20] considered the following periodic boundary value problem:

$$
\begin{aligned}
& -\left(\varphi_{p}\left(x^{\prime}\right)\right)^{\prime}+q(t) \varphi_{p}(x)=\mu a(t) \varphi_{p}(x)+g(t, x, \mu), \quad 0<t<T, \\
& x(0)=x(T), \quad x^{\prime}(0)=x^{\prime}(T) .
\end{aligned}
$$

Furthermore, if $g:[0, T] \times \mathbb{R}^{2} \rightarrow \mathbb{R}$ is continuous satisfying $g(t, x, 0) \equiv 0$, and

$$
\lim _{x \rightarrow 0} \frac{g(t, x, \lambda)}{|x|^{p-1}}=0
$$

uniformly on $[0, T]$ and $\mu$ on bounded sets. From Dancer [3], one found that problem (1.2) has two distinct unbounded sub-continua $\mathscr{C}^{+}$and $\mathscr{C}^{-}$, consisting of the bifurcation branch $\mathscr{C}$ emanating from $\left(\lambda_{1}, 0\right)$, which satisfy the following lemma.

Lemma 1.1 Either $\mathscr{C}^{+}$and $\mathscr{C}^{-}$are both unbounded and

$$
\mathscr{C}^{v} \subset\left(\left(\mathbb{R} \times P^{v}\right) \cup\left\{\left(\lambda_{1}, 0\right)\right\}\right)
$$

where $v \in\{+,-\}$.

However, among the above papers, the nonlinearities are linear in the zeros and infinity. As the problems involving non-differentiable nonlinearities, we mention the papers of Berestycki [21], Schmitt and Smith [22], Ma and Dai [23], Dai [24], Dai and Ma [25-27] and references therein. Their main tool is the bifurcation techniques. For the abstract unilateral global bifurcation theory, we refer the reader to $[1-3,20,21,23,26,28,29]$ and the references therein.

First of all, the main purpose of this paper is to establish a result similar to that of Theorem 1.1 in [26] about the Dancer-type unilateral global bifurcation result from an interval for the following periodic $p$-Laplacian problems:

$$
\begin{aligned}
& -\left(\varphi_{p}\left(x^{\prime}\right)\right)^{\prime}+q(t) \varphi_{p}(x)=\lambda a(t) \varphi_{p}(x)+F(t, x, \lambda), \quad 0<t<T, \\
& x(0)=x(T), \quad x^{\prime}(0)=x^{\prime}(T),
\end{aligned}
$$

where $1<p<+\infty, \varphi_{p}(s)=|s|^{p-2} s, \lambda$ is a positive parameter and $a(t)$ and $q(t)$ satisfy:

(H1) $a(t) \in C([0, T],(0, \infty))$.

(H2) $q \in C([0, T],[0, \infty))$ with $q(t) \not \equiv 0$. 
Meanwhile, the nonlinear term $F$ has the form $F=f+g$, where $f$ and $g$ are continuous functions on $[0, T] \times \mathbb{R}^{2}$, satisfying the following conditions:

(H3) $\left|\frac{f(t, x, \lambda)}{\varphi_{p}(x)}\right| \leq M_{1}, \forall t \in[0, T], 0<|x| \leq 1$ and all $\lambda \in \mathbb{R}$, where $M_{1}$ is a positive constant.

(H4) $g(t, x, \lambda)=o\left(|x|^{p-1}\right)$ near $x=0$ uniformly in $t \in[0, T]$ and $\lambda$ on bounded sets.

Furthermore, applying the above interval bifurcation result (Theorem 3.1), by an argument similar to that of [26], we can obtain Theorem 4.1 and Theorem 5.1 for problem (4.1) and problem (5.1).

The rest of this paper is arranged as follows. In Section 2, we given some preliminaries. In Section 3, we establish the unilateral global bifurcation result from the interval for problem (1.4). In Section 4, on the basis of the unilateral global interval bifurcation result, we shall prove the existence of the principal eigenvalues for a class of the half-quasilinear periodic $p$-Laplacian eigenvalue problem (see Theorem 4.1). In Section 5, following the above eigenvalue theory (see Theorem 4.1), we shall investigate the existence of one-sign solutions for a class of the half-quasilinear periodic $p$-Laplacian problems.

\section{Preliminaries}

Let $Y=C[0, T]$ with the norm $\|x\|_{\infty}=\max _{t \in[0, T]}|x(t)|$. Let $E=\left\{x \in C^{1}[0, T] \mid x(0)=\right.$ $\left.x(T), x^{\prime}(0)=x^{\prime}(T)\right\}$ with the norm $\|x\|=\max _{t \in[0, T]}|x(t)|+\max _{t \in[0, T]}\left|x^{\prime}(t)\right|$.

We define the linear operator $L: D(L) \subset E \rightarrow Y$,

$$
L x=-\left(\varphi_{p}\left(x^{\prime}\right)\right)^{\prime}+q(t) \varphi_{p}(x), \quad x \in D(L)
$$

with $D(L)=\left\{x, \varphi_{p}\left(x^{\prime}\right) \in C^{1}[0, T] \mid x(0)=x(T), x^{\prime}(0)=x^{\prime}(T)\right\}$. Then $L$ is a closed operator and $L^{-1}: Y \rightarrow E$ is completely continuous.

Let $P^{+}=\{x \in E \mid x(t)>0, t \in[0, T]\}$ and $P^{-}=-P^{+}$, and $P=P^{+} \cup P^{-}$. They are disjoint and open in $E$. Let $\mathscr{S}$ denote the closure in $\mathbb{R} \times E$ of the set of nontrivial solutions of (1.4),and $\mathscr{S}^{ \pm}$to denote the subset of $\mathscr{S}$ with $x \in P^{ \pm}$and $\mathscr{S}^{ \pm}=\mathscr{S}^{+} \cup \mathscr{S}^{-}$.

The following result is a generalization of Theorem 2.3 and Theorem 3.1 of [20].

Lemma 2.1 Let (H1) and (H2) hold. The linear eigenvalue problem

$$
\begin{aligned}
& -\left(\varphi_{p}\left(x^{\prime}\right)\right)^{\prime}+q(t) \varphi_{p}(x)=\lambda a(t) \varphi_{p}(x), \quad 0<t<T, \\
& x(0)=x(T), \quad x^{\prime}(0)=x^{\prime}(T),
\end{aligned}
$$

has a sequence of positive eigenvalues

$$
0<\lambda_{1} \leq \lambda_{2} \leq \lambda_{3} \leq \cdots
$$

Moreover, the eigenvalue $\lambda_{1}$ has the following properties.

(1) If $0<\lambda<\lambda_{1}$ then problem (2.1) has no nontrivial solution.

(2) $\lambda_{1}$ is a simple eigenvalue and the eigenfunctions associated to $\lambda_{1}$ is either positive or negative on $[0, T]$.

(3) Any eigenfunction $x$ associated to $\lambda \neq \lambda_{1}$ changes sign.

Lemma 2.2 (see [20]) If $(\lambda, x)$ is a nontrivial solution of (1.4) under assumptions (H1), (H2), (H3), and (H4) and $x$ has a double zero, then $x \equiv 0$. 
Next, we give an important lemma which will be used later.

Lemma 2.3 (see [30,31]) Let $b_{1}(t), b_{2}(t) \in L^{1}(I)$, I an interval and if $y$ and $z$ are functions such that $y, z, \varphi_{p}\left(y^{\prime}\right)$, and $\varphi_{p}\left(z^{\prime}\right)$ are differentiable on $I$ and $z(t) \neq 0$ for $t \in I$. Then we have the following identity.

$$
\begin{aligned}
\frac{d}{d t} & \left\{\frac{y}{\varphi_{p}(z)}\left[\varphi_{p}(z) \varphi_{p}\left(y^{\prime}\right)-\varphi_{p}(y) \varphi_{p}\left(z^{\prime}\right)\right]\right\} \\
= & \left(b_{2}-b_{1}\right)|y|^{p}+\frac{y}{\varphi_{p}(z)}\left[\varphi_{p}(z) l_{p}[y]-\varphi_{p}(y) L_{p}[z]\right] \\
& +\left[\left|y^{\prime}\right|^{p}+(p-1)\left|\frac{y z^{\prime}}{z}\right|^{p}-p \varphi_{p}(y) y^{\prime} \varphi_{p}\left(\frac{z^{\prime}}{z}\right)\right],
\end{aligned}
$$

where $1<p<+\infty, \varphi_{p}(s)=|s|^{p-2} s, l_{p}[y]=\left(\varphi_{p}\left(y^{\prime}\right)\right)^{\prime}+b_{1}(t) \varphi_{p}(y), L_{p}[z]=\left(\varphi_{p}\left(z^{\prime}\right)\right)^{\prime}+b_{2}(t) \varphi_{p}(z)$.

Remark 2.1 (see [31]) By Young's inequality, we get

$$
\left|y^{\prime}\right|^{p}+(p-1)\left|\frac{y z^{\prime}}{z}\right|^{p}-p \varphi_{p}(y) y^{\prime} \varphi_{p}\left(\frac{z^{\prime}}{z}\right) \geq 0
$$

and the equality holds if and only if $\operatorname{sgn} y=\operatorname{sgn} z$ and $\left|\frac{y^{\prime}}{y}\right|^{p}=\left|\frac{z^{\prime}}{z}\right|^{p}$.

In order to treat the problems with non-asymptotic nonlinearity at 0 and $\infty$, we shall need the following definition and lemma.

Definition 2.1 (see [32]) Let $X$ be a Banach space and let $\left\{C_{n} \mid n=1,2, \ldots\right\}$ be a certain infinite collection of subsets of $X$. Then the superior limit $\mathbb{D}$ of $\left\{C_{n}\right\}$ is defined by

$$
\mathbb{D}:=\limsup _{n \rightarrow \infty} C_{n}=\left\{x \in X \mid \exists\left\{n_{i}\right\} \subset \mathbb{N} \text { and } x_{n_{i}} \in C_{n_{i}} \text {, such that } x_{n_{i}} \rightarrow x\right\} .
$$

Lemma 2.4 (see [32]) Each connected subset of metric space $X$ is contained in a component, and each component of $X$ is closed.

Lemma 2.5 (see [33]) Let $X$ be a Banach space and let $\left\{C_{n} \mid n=1,2, \ldots\right\}$ be a family of closed connected subsets of $X$. Assume that

(i) there exist $z_{n} \in C_{n}, n=1,2, \ldots$, and $z^{*} \in X$, such that $z_{n} \rightarrow z^{*}$;

(ii) $r_{n}=\sup \left\{\|x\| \mid x \in C_{n}\right\}=\infty$;

(iii) for every $R>0,\left(\bigcup_{n=1}^{\infty} C_{n}\right) \cap B_{R}$ is a relatively compact set of $X$, where

$$
B_{R}=\{x \in X \mid\|x\| \leq R\} .
$$

Then there exists an unbounded component $C$ in $\mathbb{D}$ and $z^{*} \in C$.

\section{Unilateral global bifurcation}

We consider the following auxiliary problem (see [20], pp.2852-2855):

$$
\begin{aligned}
& -\left(\varphi_{p}\left(x^{\prime}\right)\right)^{\prime}+q(t) \varphi_{p}(x)=h(t), \quad \text { a.e. } t \in(0, T), \\
& x(0)=x(T), \quad x^{\prime}(0)=x^{\prime}(T)
\end{aligned}
$$


for a given $h \in L^{1}(0, T)$. From [20], pp.2852-2855, we see that problem (3.1) can be equivalently written as $x=G p(h)(t)$. Gp $: L^{1}(0, T) \rightarrow E$ is continuous and maps equi-integrable sets of $L^{1}(0, T)$ into relatively compacts of $E$.

Define the Nemitskii operator $H: \mathbb{R} \times E \rightarrow L^{1}(0, T)$ by

$$
H(\lambda, x)(t):=\lambda a(t) \varphi_{p}(x)+F(t, x(t), \lambda) .
$$

Then it is clear that $H$ is continuous operator and problem (1.4) can be equivalently written as

$$
x=G p \circ H(\lambda, x):=F_{\lambda}(x) .
$$

Since $G p: L^{1}(0, T) \rightarrow E$ is compact, $F_{\lambda}: \mathbb{R} \times E \rightarrow E$ is completely continuous. Obviously, $F_{\lambda}(0)=0, \forall \lambda \in \mathbb{R}$.

By an argument similar to Theorem 1.1 of [26], we can obtain the following theorem.

Theorem 3.1 Let (H1), (H2), (H3), and (H4) hold. Let $d_{1}=M_{1} / a_{0}$, where $a_{0}=$ $\min _{t \in[0, T]} a(t)$, and let $I=\left[\lambda_{1}-d_{1}, \lambda_{1}+d_{1}\right]$. The component $\mathscr{D}^{v}$ of $\mathscr{S}^{v} \cup(I \times\{0\})$, containing $I \times\{0\}$ is unbounded and lies in $\left(\mathbb{R} \times P^{v}\right) \cup(I \times\{0\})$, for $v=+$ and $v=-$. Here $\lambda_{1}$ is the principal eigenvalue of $(2.1)$.

Remark 3.1 If $F(t, x, \lambda) \equiv 0, I=\left\{\lambda_{1}\right\}$ in Theorem 3.1. Thus, a nontrivial solution of problem (1.4) does not change its sign corresponding to $\lambda_{1}$. In the present form, Theorem 3.1 is valid.

In order to prove Theorem 3.1, we need the following results.

Lemma 3.1 If $\mathscr{D}^{v}$ is bounded, we can find a neighborhood $\mathcal{O}$ of $\mathscr{D}^{v}$ such that $\partial \mathcal{O} \cap \mathscr{S}^{v}=\emptyset$, where $\mathscr{D}^{v}(v=+,-)$ is given by Theorem 3.1.

Proof We only prove the case of $\mathscr{D}_{k}^{+}$since the case of $\mathscr{D}_{k}^{-}$is similar.

Let $\mathcal{U}$ be a uniform neighborhood of $\mathscr{D}^{+}$in $\mathbb{R} \times E$.

We discuss two cases.

Case 1. If $\partial \mathcal{U} \cap \mathscr{S}^{+} \neq \emptyset$.

Since the solutions of problem (1.4) are bounded in $\mathbb{R} \times E$, then $\mathscr{D}^{+}$is compact in $\mathbb{R} \times P^{+}$. It follows that $\overline{\mathcal{U}} \cap \mathscr{S}^{+}$is compact metric space. Obviously, $\mathscr{D}^{+}$and $\partial \mathcal{U} \cap \mathscr{S}^{+}$are two disjoint closed subsets of $\overline{\mathcal{U}} \cap \mathscr{S}^{+}$. Because of the maximal connectedness of $\mathscr{D}^{+}$, there does not exist a component $\mathscr{D}^{*}$ of $\overline{\mathcal{U}} \cap \mathscr{S}^{+}$such that $\mathscr{D}^{*} \cap D^{+} \neq \emptyset, D^{*} \cap\left(\partial \mathcal{U} \cap \mathscr{S}^{+}\right) \neq \emptyset$. By [32] or [2], Lemma 1.1, there exist two disjoint compact subsets $K_{1}, K_{2}$ of $\overline{\mathcal{U}} \cap \mathscr{S}^{+}$, such that $\overline{\mathcal{U}} \cap \mathscr{S}^{+}=K_{1} \cup K_{2}, \mathscr{D}^{+} \subset K_{1}, \partial \mathcal{U} \cap \mathscr{S}^{+} \subset K_{2}$. Evidently, $d\left(K_{1}, K_{2}\right)>0$. Let $r=$ $\min \left\{d\left(K_{1}, K_{2}\right), d\left(K_{1}, \partial \mathcal{U}\right)\right\}$, and let $\mathcal{V}$ be the $\frac{r}{2}$-neighborhood of $K_{1}$. Set

$$
\mathcal{O}=\mathcal{U} \cap \mathcal{V}
$$

then

$$
\mathscr{D}^{+} \subset \mathcal{O}, \quad \partial \mathcal{O} \cap \mathscr{S}^{+}=\emptyset
$$

Case 2. If $\partial \mathcal{U} \cap \mathscr{S}^{+}=\emptyset$.

In this case, we take $\mathcal{O}=\mathcal{U}$. It is obvious that the result holds. 
To prove Theorem 3.1, we introduce the following auxiliary approximate problem:

$$
\begin{aligned}
& -\left(\varphi_{p}\left(x^{\prime}\right)\right)^{\prime}+q(t) \varphi_{p}(x)=\lambda a(t) \varphi_{p}(x)+f\left(t, x|x|^{\epsilon}, \lambda\right)+g(t, x, \lambda), \quad 0<t<T, \\
& x(0)=x(T), \quad x^{\prime}(0)=x^{\prime}(T) .
\end{aligned}
$$

Let

$$
\widehat{L} x=-L x=\left(\varphi_{p}\left(x^{\prime}\right)\right)^{\prime}-q(t) \varphi_{p}(x), \quad x \in D(L) .
$$

To prove Theorem 3.1, the next lemma will play a key role.

Lemma 3.2 Let $\epsilon_{n}, 0<\epsilon_{n}<1$, be a sequence converging to 0 . If there exists a sequence $\left(\lambda_{n}, x_{n}^{\nu}\right) \in \mathbb{R} \times P^{v}$ such that $\left(\lambda_{n}, x_{n}^{\nu}\right)$ is a nontrivial solution of problem (3.2) corresponding to $\epsilon=\epsilon_{n}$, and $\left(\lambda_{n}, x_{n}^{\nu}\right)$ converges to $(\lambda, 0)$ in $\mathbb{R} \times E$, then $\lambda \in I$.

Proof By $\left\|x_{n}^{v}\right\|^{p-1}$ for the two sides of (3.2), let $y_{n}^{v}=x_{n}^{v} /\left\|x_{n}^{v}\right\|$, then $y_{n}^{v}$ satisfies the problem

$$
\begin{aligned}
& -\left(\varphi_{p}\left(\left(y_{n}^{\nu}\right)^{\prime}\right)\right)^{\prime}+q(t) \varphi_{p}\left(y_{n}^{v}\right)=\lambda a(t) \varphi_{p}\left(y_{n}^{\nu}\right)+f_{n}(t)+g_{n}(t), \quad 0<t<T, \\
& y_{n}^{\nu}(0)=y_{n}^{v}(T), \quad\left(y_{n}^{\nu}\right)^{\prime}(0)=\left(y_{n}^{\nu}\right)^{\prime}(T),
\end{aligned}
$$

where

$$
f_{n}(t)=\frac{f\left(t, x_{n}^{\nu}\left|x_{n}^{\nu}\right|^{\epsilon_{n}}, \lambda_{n}\right)}{\left\|x_{n}^{\nu}\right\|^{p-1}}, \quad g_{n}(t)=\frac{g\left(t, x_{n}^{\nu}, \lambda_{n}\right)}{\left\|x_{n}^{\nu}\right\|^{p-1}} .
$$

Let

$$
\bar{g}(t, x, \lambda)=\max _{0 \leq|s| \leq x}|g(t, s, \lambda)| \quad \text { for all } t \in[0, T] \text { and } \lambda \text { on bounded sets, }
$$

then $\bar{g}$ is nondecreasing with respect to $x$ and

$$
\lim _{x \rightarrow 0^{+}} \frac{\bar{g}(t, x, \lambda)}{x^{p-1}}=0
$$

uniformly for $t \in[0, T]$ and $\lambda$ on bounded sets. Further it follows from (3.4) that

$$
\left|\frac{|g(t, x, \lambda)|}{\|x\|^{p-1}}\right| \leq \frac{\bar{g}(t,|x|, \lambda)}{\|x\|^{p-1}} \leq \frac{\bar{g}\left(t,\|x\|_{\infty}, \lambda\right)}{\|x\|^{p-1}} \leq \frac{\bar{g}(t,\|x\|, \lambda)}{\|x\|^{p-1}} \rightarrow 0 \quad \text { as }\|x\| \rightarrow 0
$$

uniformly for $t \in[0, T]$ and $\lambda$ on bounded sets. It follows that

$$
\lim _{n \rightarrow \infty} g_{n}(t)=0 .
$$

Clearly, (H3) implies that

$$
\begin{aligned}
\left|f_{n}(t)\right| & =\left|\frac{\left|f\left(t, x_{n}^{\nu}\left|x_{n}^{\nu}\right|^{\epsilon_{n}}, \lambda_{n}\right)\right|}{\| x_{n}^{\nu}||^{p-1} \varphi_{p}\left(y_{n}^{\nu}\right)} \cdot \varphi_{p}\left(y_{n}^{\nu}\right)\right|=\left|\frac{\left|f\left(t, x_{n}^{\nu}\left|x_{n}^{\nu}\right|^{\epsilon_{n}}, \lambda_{n}\right)\right|}{\varphi_{p}\left(x_{n}^{\nu}\left|x_{n}^{v}\right|^{\epsilon_{n}}\right)} \cdot \varphi_{p}\left(\left|x_{n}^{\nu}\right|^{\epsilon_{n}}\right) \varphi_{p}\left(y_{n}^{v}\right)\right| \\
& \leq\left.\left.\left|M_{1} \cdot\right| x_{n}^{v}\right|^{(p-1) \epsilon_{n}} \cdot \varphi_{p}\left(y_{n}^{\nu}\right)\left|\leq M_{1} \cdot\right| y_{n}^{\nu}\right|^{(p-1)}
\end{aligned}
$$

for all $t \in[0, T]$. 
It is obvious that (3.3), (3.5), and (3.6) imply that $y_{n}^{v}$ is bounded in $C^{2}$. By the ArzelàAscoli theorem, we may assume that $y_{n}^{v} \rightarrow y^{v}$ in $E$ with $\left\|y^{v}\right\|=1$. Clearly, we have $y^{v} \in \bar{P}^{v}$.

We claim that $y^{v} \in P^{v}$. On the contrary, suppose that $y^{v} \in \partial P^{v}$, by Lemma $2.2, y^{v} \equiv 0$, which is a contradiction with $\left\|y^{v}\right\|=1$.

Now, we deduce the boundedness of $\lambda$. Let $\psi_{\nu} \in P^{v}$ be an eigenfunction of problem (2.1) corresponding to $\lambda_{1}$.

To obtain the bound on $\lambda$, we shall compare $y_{n}^{v}$ and $\psi_{v}$ via Lemma 2.3. We know that $y_{n}^{v}$ satisfies

$$
\left(\varphi_{p}\left(\left(y_{n}^{v}\right)^{\prime}\right)\right)^{\prime}-q(t) \varphi_{p}\left(y_{n}^{v}\right)=-\lambda_{n} a(t) \varphi_{p}\left(y_{n}^{v}\right)-f_{n}(t)-g_{n}(t)
$$

and $\psi_{v}$ satisfies

$$
\left(\varphi_{p}\left(\psi_{v}^{\prime}\right)\right)^{\prime}-q(t) \varphi_{p}\left(\psi_{v}\right)=-\lambda_{1} a(t) \varphi_{p}\left(\psi_{\nu}\right)
$$

We can assume without loss of generality that $v=+$. By Lemma 2.3, we have

$$
\int_{0}^{T}\left\{\frac{y_{n}^{+}}{\varphi_{p}\left(\psi_{+}\right)}\left[\varphi_{p}\left(\psi_{+}\right) \varphi_{p}\left(\left(y_{n}^{+}\right)^{\prime}\right)-\varphi_{p}\left(y_{n}^{+}\right) \varphi_{p}\left(\psi_{+}^{\prime}\right)\right]\right\}^{\prime} d t=A_{1}+B_{1}
$$

where

$$
A_{1}=\int_{0}^{T} \frac{y_{n}^{+}}{\varphi_{p}\left(\psi_{+}\right)}\left[\varphi_{p}\left(\psi_{+}\right) \widehat{L}\left[y_{n}^{+}\right]-\varphi_{p}\left(y_{n}^{+}\right) \widehat{L}\left[\psi_{+}\right]\right] d t
$$

and

$$
B_{1}=\int_{0}^{T}\left[\left|\left(y_{n}^{+}\right)^{\prime}\right|^{p}+(p-1)\left|\frac{y_{n}^{+} \psi_{+}^{\prime}}{\psi_{+}}\right|^{p}-p \varphi_{p}\left(y_{n}^{+}\right)\left(y_{n}^{+}\right)^{\prime} \varphi_{p}\left(\frac{\psi_{+}^{\prime}}{\psi_{+}}\right)\right] d t
$$

We shall prove that $\psi_{+}(0) \neq 0$ and $\psi_{+}(T) \neq 0$. If $\psi_{+}(0)=0$ or $\psi_{+}(T)=0$, then $\psi_{+}(T)=$ $\psi_{+}(0)=0$. Together with $\psi_{+}(t) \geq 0, t \in[0, T]$, it follows that $\psi_{+}^{\prime}(0) \geq 0, \psi_{+}^{\prime}(T) \leq 0$. Moreover, $\psi_{+}^{\prime}(0)=\psi_{+}^{\prime}(T)$ implies that $\psi_{+}^{\prime}(0)=\psi_{+}^{\prime}(T)=0$. Lemma 2.2 implies $\psi_{+}(t) \equiv 0$, $\forall t \in[0, T]$, which is a contradiction.

Furthermore, the left-hand side of (3.7) equals zero. Clearly, we have $B_{1} \geq 0$. It follows that

$$
\begin{aligned}
0 & \geq A_{1}=\int_{0}^{T} \frac{y_{n}^{+}}{\varphi_{p}\left(\psi_{+}\right)}\left[\varphi_{p}\left(\psi_{+}\right) \widehat{L}\left[y_{n}^{+}\right]-\varphi_{p}\left(y_{n}^{+}\right) \widehat{L}\left[\psi_{+}\right]\right] d t \\
& =\int_{0}^{T}\left(\lambda_{1}-\lambda_{n}\right) a(t)\left|y_{n}^{+}\right|^{p} d t-\int_{0}^{T} y_{n}^{+}\left[f_{n}(t)+g_{n}(t)\right] d t
\end{aligned}
$$

Similarly, we can prove that $y_{n}^{+}(0) \neq 0$ and $y_{n}^{+}(T) \neq 0$. Furthermore, we can also show

$$
\begin{aligned}
0 & \geq A_{2}=\int_{0}^{T} \frac{\psi_{+}}{\varphi_{p}\left(y_{n}^{+}\right)}\left[\varphi_{p}\left(y_{n}^{+}\right) \cdot \widehat{L}\left[\psi_{+}\right]-\varphi_{p}\left(\psi_{+}\right) \cdot \widehat{L}\left[y_{n}^{+}\right]\right] d t \\
& =\int_{0}^{T}\left(\lambda_{n}-\lambda_{1}\right) a(t)\left|\psi_{+}\right|^{p} d t+\int_{0}^{T} \frac{\left|\psi_{+}\right|^{p}}{\varphi_{p}\left(y_{n}^{+}\right)}\left[f_{n}(t)+g_{n}(t)\right] d t .
\end{aligned}
$$


If $\lambda \leq \lambda_{1}$, by (3.5), (3.6), and (3.8), we obtain

$$
\begin{aligned}
\int_{0}^{T}\left(\lambda_{1}-\lambda\right) a(t)\left|y^{+}\right|^{p} d t & \leq \lim _{n \rightarrow \infty} \int_{0}^{T} y_{n}^{+}\left[f_{n}(t)+g_{n}(t)\right] d t \\
& \leq M_{1} \int_{0}^{T}\left(y^{+}\right)^{p} d t
\end{aligned}
$$

Hence, we get

$$
\int_{0}^{T}\left(\lambda_{1}-\lambda\right) a(t)\left(y^{+}\right)^{p} d t \leq M_{1} \int_{0}^{T}\left(y^{+}\right)^{p} d t
$$

which implies $\lambda \geq \lambda_{1}-d$.

If $\lambda \geq \lambda_{1}$, by (3.5), (3.6), and (3.9), we obtain

$$
\begin{aligned}
\int_{0}^{T}\left(\lambda-\lambda_{1}\right) a(t)\left|\psi_{+}\right|^{p} d t & \leq-\lim _{n \rightarrow \infty} \int_{0}^{T} \frac{\left|\psi_{+}\right|^{p}}{\varphi_{p}\left(y_{n}^{+}\right)} f_{n}(t) d t \\
& \leq M_{1} \int_{0}^{T} \psi_{+}^{p} d t .
\end{aligned}
$$

Hence, we get

$$
\int_{0}^{T}\left(\lambda-\lambda_{1}\right) a(t) \psi_{+}^{p} d t \leq M_{1} \int_{0}^{T} \psi_{+}^{p} d t
$$

which implies $\lambda \leq \lambda_{1}+d$. Therefore, we have $\lambda \in I$.

Remark 3.2 In [26], all eigenvalues of the linear eigenvalue problem are simple. However, eigenvalues of the linear periodic eigenvalue problem (2.1) are different (see [34], p.284, L.1-6, that is, these eigenvalues may be double, and at most two distinct eigenvalues can arise from them via a linear perturbation). Thus, the method of the proof of Lemma 3.2 playing a key role in this paper is different from the method of the proof of Lemma 2.3 in [26].

Lemma 3.3 For $v \in\{+,-\}$, the component $\mathscr{D}^{v}$ of $\mathscr{S}^{v} \cup(I \times\{0\})$ satisfies $\mathscr{D}^{v} \subset\left(\mathbb{R} \times P^{v}\right) \cup$ $(I \times\{0\})$, where $\mathscr{D}^{v}(v=+,-)$ be given by Theorem 3.1 .

Proof We only prove the case of $\mathscr{D}_{k}^{+}$since the case of $\mathscr{D}_{k}^{-}$is similar.

For any $(\lambda, x) \in \mathscr{D}^{+}$, there are two possibilities: (i) $x \in P^{+}$or (ii) $x \in \partial P^{+}$. It is obvious that $(\lambda, x) \in \mathbb{R} \times P^{+}$in the case of (i). The case (ii) implies that $x \in \partial P^{+}$has at least one double zero in $[0, \mathrm{~T}]$. From Lemma 2.2 it follows that $x \equiv 0$. Hence, there exists a sequence $\left(\lambda_{n}, x_{n}\right) \in \mathbb{R} \times P^{+}$such that $\left(\lambda_{n}, x_{n}\right)$ is a solution of problem (3.2) corresponding to $\epsilon=0$, and $\left(\lambda_{n}, x_{n}\right)$ converges to $(\lambda, 0)$ in $\mathbb{R} \times P^{+}$. By Lemma 3.2, we have $\lambda \in I$, i.e., $(\lambda, x) \in I \times\{0\}$ in the case of (ii). Hence, $\mathscr{D}^{+} \subset\left(\mathbb{R} \times P^{+}\right) \cup(I \times\{0\})$.

Proof of Theorem 3.1 We only prove the case of $\mathscr{D}^{+}$since the case of $\mathscr{D}^{-}$is similar. Let $\mathscr{D}^{+}$ be the component of $\mathscr{S}^{+} \cup(I \times\{0\})$, containing $I \times\{0\}$.

By Lemma 3.3, we have $\mathscr{D}^{+} \subset\left(\mathbb{R} \times P^{+}\right) \cup(I \times\{0\})$. 
Suppose on the contrary that $\mathscr{D}^{+}$is bounded. By Lemma 3.1, we can find a neighborhood $\mathcal{O}$ of $\mathscr{D}^{+}$such that $\partial \mathcal{O} \cap \mathscr{S}^{+}=\emptyset$.

In order to complete the proof of this theorem, we consider problem (3.2). For $\epsilon>0$, it is easy to show that the nonlinear term $f\left(t, x|x|^{\epsilon}, \lambda\right)+g(t, x, \lambda)$ satisfies the condition (1.3). Let

$$
\mathscr{S}_{\epsilon}=\overline{\{(\lambda, x):(\lambda, x) \text { satisfies }(3.2) \text { and } x \neq \equiv 0}^{\mathbb{R} \times E} \text {. }
$$

By Lemma 1.1, there are two unbounded continua, $\mathscr{D}_{\epsilon}^{+}$of $\mathscr{S}_{\epsilon}^{+}$and $\mathscr{D}_{\epsilon}^{-}$of $\mathscr{S}_{\epsilon}^{-}$, bifurcating from $\left(\lambda_{1}, 0\right)$, consisting of the bifurcation branch $\mathscr{D}_{\epsilon}$, where $\mathscr{D}_{\epsilon}^{+}$and $\mathscr{D}_{\epsilon}^{-}$are both unbounded and

$$
\mathscr{D}_{\epsilon}^{v} \subset\left(\mathbb{R} \times P^{v}\right) \cup\left\{\left(\lambda_{1}, 0\right)\right\}
$$

So there exists $\left(\lambda_{\epsilon}, x_{\epsilon}\right) \in \mathscr{D}_{\epsilon}^{+} \cap \partial \mathcal{O}$ for all $\epsilon>0$. Since $\mathcal{O}$ is bounded in $\mathbb{R} \times P^{+}$, equation (3.2) shows that $\left(\lambda_{\epsilon}, x_{\epsilon}\right)$ is bounded in $\mathbb{R} \times C^{2}$ independently of $\epsilon$. By the compactness of $L^{-1}$, one can find a sequence $\epsilon_{n} \rightarrow 0$ such that $\left(\lambda_{\epsilon}, x_{\epsilon}\right)$ converges to a solution $(\lambda, x)$ of $(1.4)$. So $x \in \bar{P}^{+}$. If $x \in \partial P^{+}$, then from Lemma 2.2 it follows that $x \equiv 0$. Note that $(\lambda, x) \in \mathscr{D}_{\epsilon}^{+} \cap \partial \mathcal{O}$ since $\mathscr{D}_{\epsilon}^{+} \cap \partial \mathcal{O}$ is a closed subset of $\mathbb{R} \times P^{+}$. By Lemma 3.2, $\lambda \in I$, which contradicts the definition of $\mathcal{O}$. On the other hand, if $x \in P^{+}$, then $(\lambda, x) \in \mathscr{S}^{+} \cap \partial \mathcal{O}$, which contradicts $\mathscr{S}^{+} \cap \partial \mathcal{O}=\emptyset$.

From Theorem 3.1 and its proof, we can easily get the following two corollaries.

Corollary 3.1 There exist two unbounded sub-continua $\mathscr{D}^{+}$and $\mathscr{D}^{-}$of solutions of (1.4) in $\mathbb{R} \times E$, bifurcating from $I \times\{0\}$, and $\mathscr{D}^{v} \subset\left(\mathbb{R} \times P^{v}\right) \cup(I \times\{0\})$ for $v=+$ and $v=-$.

We relax the assumption of $a$ as follows:

(A1) $a(t) \in C[0, T]$ is a sign-changing weight.

Corollary 3.2 Let (A1) hold and $f \equiv 0$. Then there exist two unbounded sub-continua $\mathscr{C}^{+}$ and $\mathscr{C}^{-}$of solutions of (1.4) in $\mathbb{R} \times E$, bifurcating from $\left(\lambda_{1}, 0\right)$, and $\mathscr{C}^{v} \subset\left(\mathbb{R} \times P^{v}\right) \cup(I \times\{0\})$ for $v=+$ and $v=-$.

\section{Spectrum of half-quasilinear eigenvalue problems}

In this section, we consider the half-quasilinear eigenvalue problem

$$
\begin{aligned}
& -\left(\varphi_{p}\left(x^{\prime}\right)\right)^{\prime}+q(t) \varphi_{p}(x)=\lambda a(t) \varphi_{p}(x)+\alpha(t) \varphi_{p}\left(x^{+}\right)+\beta(t) \varphi_{p}\left(x^{-}\right), \quad 0<t<T, \\
& x(0)=x(T), \quad x^{\prime}(0)=x^{\prime}(T),
\end{aligned}
$$

where $x^{+}=\max \{x, 0\}, x^{-}=-\min \{x, 0\}, \alpha(t), \beta(t) \in C[0, T]$.

Problem (4.1) is called half-quasilinear because it is positive $p-1$ homogeneous in the cones $u>0$ and $u<0$. Similar to [21], we say that $\lambda$ is a half-eigenvalue of problem (4.1), if there exists a nontrivial solution $\left(\lambda, x_{\lambda}\right) . \lambda$ is also said to be simple if $y=c x_{\lambda}, c>0$ for all solutions $(\lambda, y)$ of problem (4.1). A half-eigenvalue is called the principal half-eigenvalue if the corresponding eigenfunction is positive or negative.

By an argument similar to that of [26], we can obtain the following theorem. 
Theorem 4.1 There exist two simple half-eigenvalues $\lambda^{+}$and $\lambda^{-}$for problem (4.1). The corresponding half-linear solutions are in $\left\{\lambda^{+}\right\} \times P^{+}$and $\left\{\lambda^{-}\right\} \times P^{-}$. Furthermore, aside from $\lambda^{+}$and $\lambda^{-}$, there is no other half-eigenvalue with positive or negative eigenfunction.

Proof By Theorem 3.1, we know that there exists at least one solution of problem (4.1), $\left(\lambda^{\nu}, x^{\nu}\right) \in \mathbb{R} \times P^{\nu}$, for every $v=+$ and $v=-$. The positive homogeneous of problem (4.1) implies that $\left\{\left(\lambda^{\nu}, c x^{\nu}\right), c>0\right\}$ are half-linear solutions in $\left\{\lambda^{\nu}\right\} \times P^{\nu}$. Lemma 2.2 implies that any nontrivial solution $x$ of problem (4.1) lies in some $P^{\nu}$. We claim that for any solution $(\lambda, x)$ of problem (4.1) with $x \in P^{\nu}$, we have $\lambda=\lambda^{\nu}$ and $x=c x^{\nu}$ for some positive constant $c$.

Next, we only prove the case of $v=+$ since the case of $v=-$ is similar.

Applying Lemma 2.3 to $x$ and $x^{+}$on $[0, T]$, we have

$$
\begin{aligned}
\int_{0}^{T} & {\left[x^{+} \varphi_{p}\left(\left(x^{+}\right)^{\prime}\right)-\frac{\left|x^{+}\right| \varphi_{p}\left(x^{\prime}\right)}{\varphi_{p}(x)}\right]^{\prime} d t } \\
= & \int_{0}^{T}\left[x^{+} \widehat{L}\left[x^{+}\right]-\frac{x^{+} \varphi_{p}\left(x^{+}\right) \widehat{L}[x]}{\varphi_{p}(x)}\right] d t \\
& \quad+\int_{0}^{T}\left[\left|\left(x^{+}\right)^{\prime}\right|^{p}+(p-1)\left|\frac{x^{+} x^{\prime}}{x}\right|^{p}-p \varphi_{p}\left(x^{+}\right)\left(x^{+}\right)^{\prime} \varphi_{p}\left(\frac{x^{\prime}}{x}\right)\right] d t .
\end{aligned}
$$

Using a proof similar to that of Lemma 3.2, we can obtain $\lambda^{+} \leq \lambda$. Similarly, we have $\lambda^{+} \geq \lambda$. It follows that $\lambda^{+}=\lambda$.

Next, we shall show that $x=c \lambda^{v}$ for some positive constant $c$.

Since $\lambda=\lambda^{+}$, we have

$$
\int_{0}^{T}\left[x^{+} \widehat{L}\left[x^{+}\right]-\frac{x^{+} \varphi_{p}\left(x^{+}\right)}{\varphi_{p}(x)} \widehat{L}[x]\right] d t=0
$$

Furthermore, by (4.2), we obtain

$$
\int_{0}^{T}\left[\left|\left(x^{+}\right)^{\prime}\right|^{p}+(p-1)\left|\frac{x^{+} x^{\prime}}{x}\right|^{p}-p \varphi_{p}\left(x^{+}\right)\left(x^{+}\right)^{\prime} \varphi_{p}\left(\frac{x^{\prime}}{x}\right)\right] d t=0 .
$$

By Remark 2.1, we have $\operatorname{sgn} x^{+}=\operatorname{sgn} x$ and $\left|\frac{\left(x^{+}\right)^{\prime}}{x^{+}}\right|^{p}=\left|\frac{x^{\prime}}{x}\right|^{p}$. It follows that there exists a constant $c \neq 0$ such that $x=c x^{+}$for some positive constant $c$.

Naturally, we can consider the bifurcation structure of the perturbation of problem (4.1) of the form

$$
\begin{aligned}
& -\left(\varphi_{p}\left(x^{\prime}\right)\right)^{\prime}+q(t) \varphi_{p}(x) \\
& \quad=\lambda a(t) \varphi_{p}(x)+\alpha(t) \varphi_{p}\left(x^{+}\right)+\beta(t) \varphi_{p}\left(x^{-}\right)+g(t, x, \lambda), \quad 0<t<T, \\
& x(0)=x(T), \quad x^{\prime}(0)=x^{\prime}(T),
\end{aligned}
$$

where $g$ satisfies (1.3).

Remark 4.1 By some simple computation, we can show that if $\beta \equiv 0$ then $\lambda^{-}=\lambda_{1}, \alpha \equiv 0$ implies $\lambda^{+}=\lambda_{1}$ and $\alpha=\beta \equiv 0$ implies $\lambda^{+}=\lambda^{-}=\lambda_{1}$.

Remark 4.2 Note that $\min \left\{\lambda^{+}, \lambda^{-}\right\}$is the least half-eigenvalue for (4.1). 
Using a similar method to prove ([26], Theorem 3.1) with obvious changes, we may obtain the following result.

Theorem 4.2 For $v=+,-,\left(\lambda^{\nu}, 0\right)$ is a bifurcation point for problem (4.3). Moreover, there exists an unbounded continuum $\mathscr{D}^{v}$ of solutions of problem (4.3), such that $\mathscr{D}^{v} \subset((\mathbb{R} \times$ $\left.\left.P^{v}\right) \cup\left\{\left(\lambda^{\nu}, 0\right)\right\}\right)$.

Proof Let $\alpha^{0}:=\max _{t \in[0, T]}|\alpha(t)|$ and $\beta^{0}:=\max _{t \in[0, T]}|\beta(t)|$. We have

$$
I_{0}=\left[\lambda_{1}-\frac{\alpha^{0}+\beta^{0}}{a_{0}}, \lambda_{1}+\frac{\alpha^{0}+\beta^{0}}{a_{0}}\right]
$$

Corollary 3.1 shows that there exist two unbounded sub-continua $\mathscr{D}^{+}$and $\mathscr{D}^{-}$of solutions of (4.3) in $\mathbb{R} \times E$, bifurcating from $I_{0} \times\{0\}$, and $\mathscr{D}^{v} \subset\left(\mathbb{R} \times P^{v}\right) \cup\left(I_{0} \times\{0\}\right)$ for $v=+$ and $v=-$. Let us show that $\mathscr{D}^{v} \cap(\mathbb{R} \times\{0\})=\left(\lambda^{v}, 0\right)$, i.e., $\left(\lambda^{v}, 0\right)$ is a bifurcation point for problem (4.3). Indeed, assume there exists $\left(\lambda_{n}, x_{n}\right)$, a sequence of solutions of problem (4.3) converging to $(\lambda, 0)$. By $\left\|x_{n}\right\|^{p-1}$ for the two sides of (4.3), let $y_{n}=\frac{x_{n}}{\left\|x_{n}\right\|}$, then $y_{n}$ should be a solution of the problem

$$
y_{n}=G_{p}\left[\lambda a(t) \varphi_{p}\left(y_{n}\right)+\alpha(t) \varphi_{p}\left(y_{n}^{+}\right)+\beta(t) \varphi_{p}\left(y_{n}^{-}\right)+\frac{g\left(t, x_{n}, \lambda\right)}{\left\|x_{n}\right\|^{p-1}}\right] .
$$

By (3.5) and the compactness of $G_{p}$, we obtain for some convenient subsequence $y_{n} \rightarrow y_{0}$ as $n \rightarrow+\infty$. Now $y_{0}$ verifies the equation

$$
L y_{0}=\lambda a(t) \varphi_{p}\left(y_{0}\right)+\alpha(t) \varphi_{p}\left(y_{0}^{+}\right)+\beta(t) \varphi_{p}\left(y_{0}^{-}\right)
$$

and $\left\|y_{0}\right\|_{\infty}=1$. This implies that $\lambda=\lambda^{v}$ for $v \in\{+,-\}$.

Remark 4.3 Theorem 4.2 indicates that the bifurcation interval $I=\left\{\lambda^{+}, \lambda^{-}\right\}$, i.e., problem (4.3), the bifurcation interval $I$ is a finite point set. What conditions can ensure that the component indeed bifurcates from an interval is still an open problem for the secondorder periodic boundary value problems.

\section{One-sign solutions for half-linear eigenvalue problems}

Following the above eigenvalue theory (see Theorem 4.1), we shall investigate the existence of one-sign solutions for the following periodic problem:

$$
\begin{aligned}
& -\left(\varphi_{p}\left(x^{\prime}\right)\right)^{\prime}+q(t) \varphi_{p}(x)=\alpha(t) \varphi_{p}\left(x^{+}\right)+\beta(t) \varphi_{p}\left(x^{-}\right)+r a(t) f(x), \quad 0<t<T, \\
& x(0)=x(T), \quad x^{\prime}(0)=x^{\prime}(T),
\end{aligned}
$$

where $a(t)$ and $q(t)$ satisfy the conditions (H1) and (H2), respectively. We assume that $f$ satisfies the following assumptions:

(H5) $s f(s)>0$ for $s \neq 0$.

(H6) $f_{0}, f_{\infty} \in(0, \infty)$.

(H7) $f_{0}=\infty$ and $f_{\infty} \in(0, \infty)$.

(H8) $f_{0} \in(0, \infty)$ and $f_{\infty}=\infty$. 
(H9) $f_{0}=\infty$ and $f_{\infty}=\infty$.

(H10) $f_{0}=0$ and $f_{\infty}=\infty$.

Here

$$
f_{0}=\lim _{|s| \rightarrow 0} \frac{f(s)}{\varphi_{p}(s)}, \quad f_{\infty}=\lim _{|s| \rightarrow+\infty} \frac{f(s)}{\varphi_{p}(s)} .
$$

Let $\Sigma^{v}$ denote the closure of set of those solutions of (5.1) which belong to $P^{v}$.

In the case of $\alpha=\beta=0$ and $p=2$, Ma et al. [18] considered problem (5.1) on determining the interval of $r$ by the bifurcation theory of Rabinowitz [2] and Kielhöfer [35], in which there exist positive solutions for problem (5.1) with indefinite weight under the assumptions (H6). In the case of $\alpha=\beta=0$, Dai et al. [20] have established the existence of positive solutions for the $p$-Laplacian problem (5.1) with crossing nonlinearity, which extends the results of [18].

Remark 5.1 For $p=2$ and $a(t)=1, t \in[0, T]$, by using the Prüfer methods, Binding and Rynne [34] studied the spectral theory and degree theoretic properties of (5.1). In the following, they considered the existence and non-existence of solutions of the corresponding half-linear problems.

Remark 5.2 The nonlinear term of (5.1) is not necessarily homogeneous linearizable at the origin and infinity because of the influence of the term $\alpha(t) x^{+}+\beta(t) x^{-}$. Clearly, the bifurcation results of $[1-3,18,19,28,29,35]$ cannot be applied directly to obtain our results.

Remark 5.3 Using the unilateral global bifurcation result from the interval, we can obtain some results as regards the existence of one-sign solutions which extend the corresponding results of [20] to some extent.

To state the main result of this section, we study the following eigenvalue problem:

$$
\begin{aligned}
& -\left(\varphi_{p}\left(x^{\prime}\right)\right)^{\prime}+q(t) \varphi_{p}(x)=\alpha(t) \varphi_{p}\left(x^{+}\right)+\beta(t) \varphi_{p}\left(x^{-}\right)+\lambda r a(t) f(x), \quad 0<t<T, \\
& x(0)=x(T), \quad x^{\prime}(0)=x^{\prime}(T),
\end{aligned}
$$

where $\lambda>0$ is a parameter. Let $\zeta \in C(\mathbb{R}, \mathbb{R})$ be such that

$$
f(x)=f_{0} \varphi_{p}(x)+\zeta(x)
$$

with $\lim _{|x| \rightarrow 0} \frac{\zeta(x)}{\|x\|^{p-1}}=0$. Let us consider

$$
\begin{aligned}
& -\left(\varphi_{p}\left(x^{\prime}\right)\right)^{\prime}+q(t) \varphi_{p}(x) \\
& \quad=\lambda r a(t) f_{0} \varphi_{p}(x)+\alpha(t) \varphi_{p}\left(x^{+}\right)+\beta(t) \varphi_{p}\left(x^{-}\right)+\lambda r a(t) \zeta(x), \quad t \in(0, T), \\
& x(0)=x(T), \quad x^{\prime}(0)=x^{\prime}(T)
\end{aligned}
$$

as a bifurcation problem from the trivial solution $x \equiv 0$.

Applying Theorem 4.2 to problem (5.3), we have the following result. 
Lemma 5.1 For $v=+,-,\left(\frac{\lambda^{v}}{r_{0}}, 0\right)$ is a bifurcation point for problem (5.3). Moreover, there exists an unbounded continuum $\mathscr{D}^{v}$ of solutions of problem (5.3), such that $\mathscr{D}^{v} \subset((\mathbb{R} \times$ $\left.\left.P^{v}\right) \cup\left\{\left(\frac{\lambda^{v}}{r f_{0}}, 0\right)\right\}\right)$.

By an argument similar to that of [26], we can obtain the following theorem.

Theorem 5.1 Let (H1), (H2), (H5), and (H6) hold. For $v=+$,-, either $\frac{\lambda^{v}}{f_{\infty}}<r<\frac{\lambda^{v}}{f_{0}}$ or $\frac{\lambda^{v}}{f_{0}}<$ $r<\frac{\lambda^{v}}{f_{\infty}}$. Then problem (5.1) possesses two solutions $x^{+}, x^{-}$, such that $v x^{\nu}>0$.

Remark 5.4 From (H5) and (H6), we can see that there exists a positive constant $Q$, such that $\frac{f(s)}{\varphi_{p}(s)} \geq Q$ for all $s \neq 0$.

Remark 5.5 Note that if $\alpha(t)=\beta(t) \equiv 0$ the result of Theorem 5.1 is equivalent to those of Theorem 5.1 of [20], Theorem 5.1 extends the corresponding results of [20].

In order to prove Theorem 5.1, we need the following Sturm-type comparison result.

Lemma 5.2 Let $b_{2}(t) \geq \max \left\{b_{1}(t), b_{1}(t)+\alpha+\beta, b_{1}(t)-\alpha-\beta\right\}$ for $t \in(0, T)$ and $b_{i}(t) \in$ $C(0, T), i=1,2$. Also let $u_{1}, u_{2}$ be solutions of the following differential equations:

$$
\begin{aligned}
& \left(\varphi_{p}\left(u^{\prime}\right)\right)^{\prime}-q(t) \varphi_{p}(u)+b_{i}(t) \varphi_{p}(u)+\alpha(t) \varphi_{p}\left(u^{+}\right)+\beta(t) \varphi_{p}\left(u^{-}\right)=0, \quad i=1,2, t \in(0, T), \\
& u(0)=u(T), \quad u^{\prime}(0)=u^{\prime}(T),
\end{aligned}
$$

respectively. If $(c, d) \subseteq(0, T)$, and $u_{1}(c)=u_{1}(d)=0, u_{1}(t) \neq 0$ in $(c, d)$, then either there exists $\tau \in(c, d)$ such that $u_{2}(\tau)=0$ or $b_{2}=b_{1}$ or $b_{2}=b_{1}+\alpha+\beta$ or $b_{2}=b_{1}-\alpha-\beta$ and $u_{2}(t)=\mu u_{1}(t)$ for some constant $\mu \neq 0$.

Proof We discuss four cases.

Case 1. $u_{1}(t)>0, u_{2}(t)>0$ in $(c, d)$. By Lemma 2.3, we have

$$
\begin{aligned}
\int_{c}^{d} & \left\{\frac{u_{1}}{\varphi_{p}\left(u_{2}\right)}\left[\varphi_{p}\left(u_{2}\right) \varphi_{p}\left(u_{1}^{\prime}\right)-\varphi_{p}\left(u_{1}\right) \varphi_{p}\left(u_{2}^{\prime}\right)\right]\right\}^{\prime} d t \\
= & \int_{c}^{d} \frac{u_{1}}{\varphi_{p}\left(u_{2}\right)}\left[\varphi_{p}\left(u_{2}\right) \widehat{L}\left[u_{1}\right]-\varphi_{p}\left(u_{1}\right) \widehat{L}\left[u_{2}\right]\right] d t \\
& +\int_{c}^{d}\left[\left|u_{1}^{\prime}\right|^{p}+(p-1)\left|\frac{u_{1} u_{2}^{\prime}}{u_{2}}\right|^{p}-p \varphi_{p}\left(u_{1}\right) u_{1}^{\prime} \varphi_{p}\left(\frac{u_{2}^{\prime}}{u_{2}}\right)\right] d t .
\end{aligned}
$$

It follows that

$$
\begin{aligned}
& \int_{c}^{d}\left[u_{1} \varphi_{p}\left(u_{1}\right)^{\prime}-\frac{u_{1}^{p} \varphi_{p}\left(u_{2}^{\prime}\right)}{\varphi_{p}\left(u_{2}\right)}\right]^{\prime} d t \\
& \quad=\int_{c}^{d}\left\{\left(b_{2}-b_{1}\right)\left|u_{1}\right|^{p}+\left[\left|u_{1}^{\prime}\right|^{p}+(p-1)\left|\frac{u_{1} u_{2}^{\prime}}{u_{2}}\right|^{p}-p \varphi_{p}\left(u_{1}\right) u_{1}^{\prime} \varphi_{p}\left(\frac{u_{2}^{\prime}}{u_{2}}\right)\right]\right\} d t .
\end{aligned}
$$

The left-hand side of (5.4) equals

$$
\lim _{t \rightarrow c^{+}} \frac{u_{1}^{p} \varphi_{p}\left(u_{2}^{\prime}\right)}{\varphi_{p}\left(u_{2}\right)}-\lim _{t \rightarrow d^{-}} \frac{u_{1}^{p} \varphi_{p}\left(u_{2}^{\prime}\right)}{\varphi_{p}\left(u_{2}\right)}=H_{c}-H_{d}
$$


We prove that $H_{c}=0$. If $u_{2}(c) \neq 0$, then $H_{c}=0$. If $u_{2}(c)=0$, noting the conclusion of Lemma 2.2, then $u_{2}^{\prime}(c)>0$. By the L'Hospital rule, we have

$$
\begin{aligned}
H_{c} & =\lim _{t \rightarrow c^{+}} \frac{u_{1}^{p} \varphi_{p}\left(u_{2}^{\prime}\right)}{\varphi_{p}\left(u_{2}\right)}=\lim _{t \rightarrow c^{+}} \frac{p u_{1}^{p-1} u_{1}^{\prime} \varphi_{p}\left(u_{2}^{\prime}\right)+u_{1}^{p}\left(\varphi_{p}\left(u_{2}^{\prime}\right)\right)^{\prime}}{(p-1) u_{2}^{p-2} u_{2}^{\prime}} \\
& =\lim _{t \rightarrow c^{+}} \frac{p u_{1}^{p-1} u_{1}^{\prime} \varphi_{p}\left(u_{2}^{\prime}\right)-u_{1}^{p} \cdot\left[q(t) \varphi_{p}\left(u_{2}\right)-b_{2}(t) \varphi_{p}\left(u_{2}\right)-\alpha \varphi_{p}\left(u_{2}^{+}\right)-\beta \varphi_{p}\left(u_{2}^{-}\right)\right]}{(p-1) u_{2}^{p-2} u_{2}^{\prime}} \\
& =\lim _{t \rightarrow c^{+}} \frac{p u_{1}^{p-1} u_{1}^{\prime} \varphi_{p}\left(u_{2}^{\prime}\right)}{(p-1) u_{2}^{p-2} u_{2}^{\prime}}-\lim _{t \rightarrow c^{+}} \frac{u_{1}^{p} \cdot\left[q(t) u_{2}^{p-1}-b_{2}(t) u_{2}^{p-1}-\alpha u_{2}^{p-1}\right]}{(p-1) u_{2}^{p-2} u_{2}^{\prime}} \\
& =\lim _{t \rightarrow c^{+}} \frac{p u_{1}^{p-1} u_{1}^{\prime} \varphi_{p}\left(u_{2}^{\prime}\right)}{(p-1) u_{2}^{p-2} u_{2}^{\prime}}=\frac{p u_{1}^{\prime}(c)\left|u_{2}^{\prime}(c)\right|^{p-2}}{p-1} \cdot \lim _{t \rightarrow c^{+}} \frac{u_{1}^{p-1}}{u_{2}^{p-2}} .
\end{aligned}
$$

If $p \leq 2$, then $H_{c}=0$. If $2<p \leq 3$, applying the L'Hospital rule again, we obtain

$$
\lim _{t \rightarrow c^{+}} \frac{u_{1}^{p-1}}{u_{2}^{p-2}}=\frac{(p-1) u_{1}^{\prime}(c)}{(p-2) u_{2}^{\prime}(c)} \lim _{t \rightarrow c^{+}} \frac{u_{1}^{p-2}}{u_{2}^{p-3}} .
$$

This implies that $H_{c}=0$. If $k<p \leq k+1$, then we continue with this process $k$ times to obtain $H_{c}=0$.

Similarly, we can obtain $H_{d}=0$. Therefore, the left-hand side of (5.4) equals zero. Hence, the right-hand side of (5.4) also equals zero.

By Remark 2.1, we have $\operatorname{sgn} u_{1}=\operatorname{sgn} u_{2}$ and $\left|\frac{u_{1}^{\prime}}{u_{1}}\right|^{p}=\left|\frac{u_{2}^{\prime}}{u_{2}}\right|^{p}$. It follows that there exists a constant $\mu \neq 0$ such that $u_{2}=\mu u_{1}$ and $b_{2}=b_{1}$.

Case 2. $u_{1}(t)>0, u_{2}(t)<0$. Similar to (5.4), we can get

$$
\begin{aligned}
& \int_{c}^{d}\left[u_{1} \varphi_{p}\left(u_{1}^{\prime}\right)-\frac{u_{1}^{p} \varphi_{p}\left(u_{2}^{\prime}\right)}{\varphi_{p}\left(u_{2}\right)}\right]^{\prime} d t \\
& \quad=\int_{c}^{d}\left\{\left(b_{2}-b_{1}-\alpha-\beta\right)\left|u_{1}\right|^{p}+\left[\left|u_{1}^{\prime}\right|^{p}+(p-1)\left|\frac{u_{1} u_{2}^{\prime}}{u_{2}}\right|^{p}-p u_{1}^{\prime} \varphi_{p}\left(u_{1}\right) \varphi_{p}\left(\frac{u_{2}^{\prime}}{u_{2}}\right)\right]\right\} d t .
\end{aligned}
$$

The above argument is still valid for this case.

Case 3. $u_{1}(t)<0, u_{2}(t)<0$. Similar to Case 1 , we can get the result.

Case 4. $u_{1}(t)<0, u_{2}(t)>0$. Similar to (5.4), we can get

$$
\begin{aligned}
& \int_{c}^{d}\left[u_{1} \varphi_{p}\left(u_{1}^{\prime}\right)-\frac{u_{1}^{p} \varphi_{p}\left(u_{2}^{\prime}\right)}{\varphi_{p}\left(u_{2}\right)}\right]^{\prime} d t \\
& \quad=\int_{c}^{d}\left\{\left(b_{2}-b_{1}+\alpha+\beta\right)\left|u_{1}\right|^{p}+\left[\left|u_{1}^{\prime}\right|^{p}+(p-1)\left|\frac{u_{1} u_{2}^{\prime}}{u_{2}}\right|^{p}-p u_{1}^{\prime} \varphi_{p}\left(u_{1}\right) \varphi_{p}\left(\frac{u_{2}^{\prime}}{u_{2}}\right)\right]\right\} d t .
\end{aligned}
$$

Similar to the proof of Case 1, we can obtain the result.

By Lemma 5.2, we obtain the following result, which will be used later.

Lemma 5.3 Assume $(\mathrm{H} 1)$ and $(\mathrm{H} 2)$ hold. Let $g_{n}(t) \in C([0, T],(0,+\infty))$ such that

$$
\lim _{n \rightarrow+\infty} g_{n}(t)=+\infty \quad \text { uniformly on }(0, T) \text {. }
$$


Let $y_{n}$ be a solution of the equation

$$
\left\{\begin{array}{l}
\left(\varphi_{p}\left(y_{n}^{\prime}\right)\right)^{\prime}-q(t) \varphi_{p}\left(y_{n}\right)+g_{n}(t) \varphi_{p}\left(y_{n}\right)+\alpha(t) \varphi_{p}\left(y_{n}^{+}\right)+\beta(t) \varphi_{p}\left(y_{n}^{-}\right)=0, \quad 0<t<T, \\
y_{n}(0)=y_{n}(T), \quad y_{n}^{\prime}(0)=y_{n}^{\prime}(T)=0 .
\end{array}\right.
$$

Then $y_{n}$ must change sign on $(0, T)$ as $n \rightarrow+\infty$.

Proof Set $\alpha^{0}:=\max _{t \in[0, T]}|\alpha(t)|$ and $\beta^{0}:=\max _{t \in[0, T]}|\beta(t)|$. By simple computation, we can show that

$$
g_{n}(t)+\alpha(t) \varphi_{p}\left(\frac{y_{n}^{+}}{y_{n}}\right)+\beta(t) \varphi_{p}\left(\frac{y_{n}^{-}}{y_{n}}\right) \geq g_{n}(t)-\alpha^{0}-\beta^{0} \quad \text { for all } t \in[0, T] .
$$

After taking a subsequence if necessary, we may assume that

$$
g_{n_{j}}(t)-\alpha^{0}-\beta^{0} \geq j \quad \text { for all } t \in[0, T]
$$

as $j \rightarrow+\infty$. By Lemma 2.1 , we see that any nontrivial solution $x$ of the equation

$$
\begin{aligned}
& \left(\varphi_{p}\left(x^{\prime}(t)\right)\right)^{\prime}-q(t) \varphi_{p}(x(t))+j \varphi_{p}(x(t))=0, \quad t \in(0, T), \\
& x(0)=x(T), \quad x^{\prime}(0)=x^{\prime}(T),
\end{aligned}
$$

must change sign on $(0, T)$ as $j \rightarrow+\infty$. Note that the conclusion of Lemma 5.2 also is valid if $\alpha=\beta=0$. Using these facts and Lemma 5.2, we can see that $y_{n}$ must change sign on $(0, T)$ as $n \rightarrow+\infty$.

Proof of Theorem 5.1 It is clear that any solution of $(5.2)$ of the form $(1, x)$ yields solutions $x$ of (5.1). We will show that $\mathscr{D}^{v}$ crosses the hyperplane $\{1\} \times E$. To do this, it is enough to show that $\mathscr{D}^{v}$ joins $\left(\frac{\lambda^{v}}{r f_{0}}, 0\right)$ to $\left(\frac{\lambda^{v}}{r f_{\infty}}, \infty\right)$. Let $\left(\mu_{n}, x_{n}\right) \in \mathscr{D}^{v} \backslash\left\{\left(\frac{\lambda^{v}}{r f_{0}}, 0\right)\right\}$ satisfy

$$
\mu_{n}+\left\|x_{n}\right\| \rightarrow \infty
$$

Case 1. $\frac{\lambda^{v}}{f_{\infty}}<r<\frac{\lambda^{v}}{f_{0}}$.

In this case, we show that

$$
\left(\frac{\lambda^{v}}{r f_{\infty}}, \frac{\lambda^{v}}{r f_{0}}\right) \subseteq\left\{\lambda \in \mathbb{R} \mid(\lambda, x) \in \mathscr{D}^{v}\right\}
$$

We divide the proof into two steps.

Step 1 . We show that if there exists a constant number $M>0$ such that

$$
\mu_{n} \in(0, M]
$$

then $\mathscr{D}^{v}$ joins $\left(\frac{\lambda^{v}}{r f_{0}}, 0\right)$ to $\left(\frac{\lambda^{v}}{r f_{\infty}}, \infty\right)$.

In this case, it follows that

$$
\left\|x_{n}\right\| \rightarrow+\infty
$$


Let $\xi \in C(\mathbb{R}, \mathbb{R})$,

$$
f(x)=f_{\infty} \varphi_{p}(x)+\xi(x)
$$

Let $\bar{\xi}(x)=\max \{|\xi(s)|: 0 \leq|s| \leq x\}$, then $\bar{\xi}$ is nondecreasing and

$$
\lim _{x \rightarrow+\infty} \frac{\bar{\xi}(x)}{\varphi_{p}(x)}=0
$$

We divide the equation

$$
\begin{aligned}
& -\left(\varphi_{p}\left(x_{n}^{\prime}\right)\right)^{\prime}+q(t) \varphi_{p}\left(x_{n}\right) \\
& \quad=\mu_{n} r a(t) f_{\infty} \varphi_{p}\left(x_{n}\right)+\alpha(t) \varphi_{p}\left(x_{n}^{+}\right)+\beta(t) \varphi_{p}\left(x_{n}^{-}\right)+\mu_{n} r a(t) \xi\left(x_{n}\right), \quad t \in(0, T), \\
& x(0)=x(T), \quad x^{\prime}(0)=x^{\prime}(T),
\end{aligned}
$$

by $\left\|x_{n}\right\|^{p-1}$ and set $y_{n}=\frac{x_{n}}{\left\|x_{n}\right\|}$. Since $y_{n}$ is bounded in $C^{2}[0,1]$, choosing a subsequence and relabeling if necessary, we have $y_{n} \rightarrow y$ for some $y \in E$. Moreover, from (5.6) and the fact that $\bar{\xi}$ is nondecreasing, we have

$$
\lim _{n \rightarrow \infty} \frac{\left|\xi\left(x_{n}(t)\right)\right|}{\left\|x_{n}\right\|^{p-1}}=0, \quad \forall t \in[0, T]
$$

since

$$
\frac{\left|\xi\left(x_{n}(t)\right)\right|}{\left\|x_{n}\right\|^{p-1}} \leq \frac{\bar{\xi}\left(\left|x_{n}(t)\right|\right)}{\left\|x_{n}\right\|^{p-1}} \leq \frac{\bar{\xi}\left(\left\|x_{n}(t)\right\|_{\infty}\right)}{\left\|x_{n}\right\|^{p-1}} \leq \frac{\bar{\xi}\left(\left\|x_{n}(t)\right\|\right)}{\left\|x_{n}\right\|^{p-1}} \rightarrow 0, \quad n \rightarrow \infty, \forall t \in[0, T] .
$$

By the compactness of $L^{-1}$, we obtain

$$
\begin{aligned}
& -\left(\varphi_{p}\left(y^{\prime}\right)\right)^{\prime}+q(t) \varphi_{p}(y)=\mu r a(t) f_{\infty} \varphi_{p}(y)+\alpha(t) \varphi_{p}\left(y^{+}\right)+\beta(t) \varphi_{p}\left(y^{-}\right), \quad t \in(0, T), \\
& y(0)=y(T), \quad y^{\prime}(0)=y^{\prime}(T),
\end{aligned}
$$

where $\mu:=\lim _{n \rightarrow \infty} \mu_{n}$, again choosing a subsequence and relabeling if necessary. Thus it is clear that $\|y\|=1$ and $y \in \overline{\mathscr{D}}^{v} \subseteq \mathscr{D}^{v}$ since $\mathscr{D}^{v}$ is closed in $\mathbb{R} \times E$. Moreover, by Theorem 4.1, $\mu r f_{\infty}=\lambda^{\nu}$, so that

$$
\mu=\frac{\lambda^{\nu}}{r f_{\infty}} .
$$

Thus $\mathscr{D}^{v}$ joins $\left(\frac{\lambda^{v}}{r f_{0}}, 0\right)$ to $\left(\frac{\lambda^{v}}{r f_{\infty}}, \infty\right)$.

Step 2. We show that there exists a constant number $M>0$ such that $\mu_{n} \in(0, M]$, for all $n$.

On the contrary, we suppose that $\lim _{n+\rightarrow \infty}\left|\mu_{n}\right|=+\infty$. Since $\left(\mu_{n}, x_{n}\right) \in \mathscr{D}^{v}$, it follows from the compactness of $L^{-1}$ that

$$
\begin{aligned}
& -\left(\varphi_{p}\left(x_{n}^{\prime}\right)\right)^{\prime}+q(t) \varphi_{p}\left(x_{n}\right)=\mu_{n} r a(t) \tilde{f}_{n}(t) \varphi_{p}\left(x_{n}\right)+\alpha(t) \varphi_{p}\left(x_{n}^{+}\right)+\beta(t) \varphi_{p}\left(x_{n}^{-}\right), \quad t \in(0, T), \\
& x_{n}(0)=x_{n}(T), \quad x_{n}^{\prime}(0)=x_{n}^{\prime}(T),
\end{aligned}
$$


where $\tilde{f}_{n}:[0,1] \times[0, \infty) \rightarrow \mathbb{R}$ by

$$
\tilde{f}_{n}(t)= \begin{cases}\frac{f\left(x_{n}\right)}{\varphi_{p}\left(x_{n}\right)}, & x_{n} \neq 0, t \in[0, T], \\ f_{0}, & x_{n}=0, t \in[0, T] .\end{cases}
$$

By Remark 5.4, we have

$$
\lim _{n \rightarrow+\infty} \mu_{n} r \tilde{f}_{n}(t)= \pm \infty
$$

Let $\psi^{v}$ be an eigenfunction corresponding to $\lambda^{\nu}$. But if $\lim _{n \rightarrow+\infty} \mu_{n} r \tilde{f}_{n}(t)=-\infty$, applying Lemma 5.2 to $y_{n}$ and $\psi^{\nu}$, we see that $\psi^{v}$ must change sign for $n$ large enough, which is impossible. So $\lim _{n \rightarrow+\infty} \mu_{n} r \tilde{f}_{n}(t)=+\infty$. By Lemma 5.3, we see that $y_{n}$ must change sign for $n$ large enough, and this contradicts the fact that $y_{n} \in P^{v}$.

Case 2. $\frac{\lambda^{v}}{f_{0}}<r<\frac{\lambda^{v}}{f_{\infty}}$.

In this case, if $\left(\mu_{n}, x_{n}\right) \in \mathscr{D}^{v}$ is such that

$$
\lim _{n \rightarrow+\infty}\left(\left|\mu_{n}\right|+\left\|x_{n}\right\|\right)=\infty
$$

and

$$
\lim _{n \rightarrow+\infty}\left|\mu_{n}\right|=\infty
$$

then

$$
\left(\frac{\lambda^{v}}{r f_{0}}, \frac{\lambda^{v}}{r f_{\infty}}\right) \subseteq\left\{\lambda \in(0, \infty) \mid(\lambda, x) \in \mathscr{D}^{v}\right\}
$$

and, moreover, $(\{1\} \times E) \cap \mathscr{D}^{v} \neq \emptyset$.

Assume that there exists $M>0$ such that for all $n \in \mathbb{N}$,

$$
\left|\mu_{n}\right| \in(0, M] .
$$

Applying a similar argument to that used in Step 1 of Case 1, after taking a subsequence and relabeling if necessary, it follows that

$$
\left(\mu_{n}, x_{n}\right) \rightarrow\left(\frac{\lambda^{v}}{r f_{\infty}}, \infty\right), \quad n \rightarrow \infty
$$

Again $\mathscr{D}^{v}$ joins $\left(\frac{\lambda^{v}}{r f_{0}}, 0\right)$ to $\left(\frac{\lambda^{v}}{r f_{\infty}}, \infty\right)$ and the result follows.

Theorem 5.2 Let (H1), (H2), (H5), and (H7) hold. For $v \in\{+,-\}$, assume that one of the following conditions holds:

(i) $r \in\left(0, \frac{\lambda^{v}}{f_{\infty}}\right)$ for $\lambda^{\nu}>0$.

(ii) $r \in\left(0, \frac{\lambda^{+}}{f_{\infty}}\right) \cup\left(\frac{\lambda^{-}}{f_{\infty}}, 0\right)$ for $\nu \lambda^{\nu}>0$.

(iii) $r \in\left(0, \frac{\lambda^{-}}{f_{\infty}}\right) \cup\left(\frac{\lambda^{+}}{f_{\infty}}, 0\right)$ for $\nu \lambda^{\nu}<0$.

(iv) $r \in\left(\frac{\lambda^{v}}{f_{\infty}}, 0\right)$ for $\lambda^{v}<0$.

Then problem (5.1) possesses two solutions $x^{+}, x^{-}$, such that $v x^{v}>0$. 
Proof We shall only prove the case of (i) since the proofs of the cases for (ii), (iii), and (iv) are completely analogous.

Inspired by the idea of [36] or see [20], we define the cut-off function of $f$ as follows:

$$
f^{[n]}(s):= \begin{cases}n \varphi_{p}(s), & s \in\left[-\frac{1}{n}, \frac{1}{n}\right], \\ {\left[f\left(\frac{2}{n}\right)-\frac{1}{n^{p-2}}\right](n s-2)+f\left(\frac{2}{n}\right),} & s \in\left(\frac{1}{n}, \frac{2}{n}\right), \\ -\left[f\left(-\frac{2}{n}\right)+\frac{1}{n^{p-2}}\right](n s+2)+f\left(-\frac{2}{n}\right), & s \in\left(-\frac{2}{n}, \frac{1}{n}\right), \\ f(s), & s \in\left(-\infty,-\frac{2}{n}\right] \cup\left[\frac{2}{n},+\infty\right) .\end{cases}
$$

We consider the following problem:

$$
\begin{aligned}
& -\left(\varphi_{p}\left(x^{\prime}\right)\right)^{\prime}+q(t) \varphi_{p}(x)=\alpha(t) \varphi_{p}\left(x^{+}\right)+\beta(t) \varphi_{p}\left(x^{-}\right)+\lambda r a(t) f^{[n]}(x), \quad t \in(0, T), \\
& x(0)=x(T), \quad x^{\prime}(0)=x^{\prime}(T) .
\end{aligned}
$$

Clearly, we can see that $\lim _{n \rightarrow+\infty} f^{[n]}(s)=f(s),\left(f^{[n]}\right)_{0}=n$ and $\left(f^{[n]}\right)_{\infty}=f_{\infty}$.

Similar to the proof of Theorem 5.1, there exists an unbounded continuum $\mathscr{D}^{[n]}$ of solutions of problem (5.7) emanating from $\left(\frac{\lambda^{v}}{r n}, 0\right)$, such that $\mathscr{D}^{v[n]} \subset\left(\left(\mathbb{R} \times P^{v}\right) \cup\left\{\left(\frac{\lambda^{v}}{r n}, 0\right)\right\}\right)$, and $\mathscr{D}^{v[n]}$ joins $\left(\frac{\lambda^{v}}{r n}, 0\right)$ to $\left(\frac{\lambda^{v}}{r f_{\infty}}, \infty\right)$.

Taking $z_{n}=\left(\frac{\lambda^{\nu}}{r n}, 0\right)$ and $z^{*}=(0,0)$, we have $z_{n} \rightarrow z^{*}$.

So condition (i) in Lemma 2.5 is satisfied with $z^{*}=(0,0)$.

Obviously

$$
r_{n}=\sup \left\{\lambda+\|x\| \mid(\lambda, x) \in \mathscr{D}^{v[n]}\right\}=\infty
$$

and accordingly, (ii) in Lemma 2.5 holds. (iii) in Lemma 2.5 can be deduced directly from the Arzelà-Ascoli theorem and the definition of $f^{[n]}$.

Therefore, by Lemma $2.5, \lim \sup _{n \rightarrow \infty} \mathscr{D}^{v[n]}$ contains an unbounded component $\mathscr{D}^{v}$ with $(0,0) \in \mathscr{D}^{v}$.

From $\lim _{n \rightarrow+\infty} f^{[n]}(s)=f(s),(5.7)$ can be converted to the equivalent equation (5.2). Since $\mathscr{D}^{v[n]} \subset\left(\mathbb{R} \times P^{v}\right)$, we conclude $\mathscr{D}^{v} \subset\left(\mathbb{R} \times P^{v}\right)$. Moreover, $\mathscr{D}^{v} \subset \Sigma^{v}$ by (5.1).

Similar to the method of the proof of Case 2 of Theorem 5.1, we can obtain $\left(\frac{\lambda^{v}}{r f_{\infty}}, \infty\right) \in$ $\mathscr{D}^{v}$.

Theorem 5.3 Let (H1), (H2), (H5), and (H8) hold. For $v \in\{+,-\}$, assume that one of the following conditions holds:

(i) $r \in\left(0, \frac{\lambda^{v}}{f_{0}}\right)$ for $\lambda^{v}>0$.

(ii) $r \in\left(0, \frac{\lambda^{+}}{f_{0}}\right) \cup\left(\frac{\lambda^{-}}{f_{0}}, 0\right)$ for $\nu \lambda^{\nu}>0$.

(iii) $r \in\left(0, \frac{\lambda^{-}}{f_{0}}\right) \cup\left(\frac{\lambda^{+}}{f_{0}}, 0\right)$ for $v \lambda^{\nu}<0$.

(iv) $r \in\left(\frac{\lambda^{v}}{f_{0}}, 0\right)$ for $\lambda^{\nu}<0$.

Then problem (5.1) possesses two solutions $x^{+}, x^{-}$, such that $v x^{v}>0$.

Proof We only prove the case of (i) since the proof of (ii)-(iv) can be given similarly.

Inspired by the idea of [36] or see [20], we define the cut-off function of $f$ as follows:

$$
f^{[n]}(s):= \begin{cases}n \varphi_{p}(s), & s \in(-\infty,-2 n] \cup[2 n,+\infty), \\ \frac{n \varphi_{p}(2 n)+f(-n)}{n}(s+n)+f(-n), & s \in(-2 n,-n), \\ \frac{n \varphi_{p}(2 n)-f(n)}{n}(s-n)+f(n), & s \in(n, 2 n), \\ f(s), & s \in[-n, n] .\end{cases}
$$


We consider the following problem:

$$
\begin{aligned}
& -\left(\varphi_{p}\left(x^{\prime}\right)\right)^{\prime}+q(t) \varphi_{p}(x)=\alpha(t) \varphi_{p}\left(x^{+}\right)+\beta(t) \varphi_{p}\left(x^{-}\right)+\lambda r a(t) f^{[n]}(x), \quad t \in(0, T), \\
& x(0)=x(T), \quad x^{\prime}(0)=x^{\prime}(T) .
\end{aligned}
$$

Clearly, we can see that $\lim _{n \rightarrow+\infty} f^{[n]}(s)=f(s),\left(f^{[n]}\right)_{0}=f_{0}$, and $\left(f^{[n]}\right)_{\infty}=n$.

Similar to the proof of Theorem 5.1, there exists an unbounded continuum $\mathscr{D}^{\mathrm{r}[n]}$ of solutions of problem (5.8) emanating from $\left(\frac{\lambda^{v}}{r f_{0}}, 0\right)$, such that $\mathscr{D}^{v[n]} \subset\left(\left(\mathbb{R} \times P^{v}\right) \cup\left\{\left(\frac{\lambda^{v}}{r f_{0}}, 0\right)\right\}\right)$ and $\mathscr{D}^{v[n]}$ joins $\left(\frac{\lambda^{v}}{r f_{0}}, 0\right)$ to $\left(\frac{\lambda^{v}}{r n}, \infty\right)$.

Taking $z_{n}=\left(\frac{\lambda^{v}}{r n}, \infty\right)$ and $z^{*}=(0, \infty)$, we have $z_{n} \rightarrow z^{*}$.

So condition (i) in Lemma 2.5 is satisfied with $z^{*}=(0, \infty)$.

Obviously

$$
r_{n}=\sup \left\{\lambda+\|x\| \mid(\lambda, x) \in \mathscr{D}^{v[n]}\right\}=\infty,
$$

and accordingly, (ii) in Lemma 2.5 holds. (iii) in Lemma 2.5 can be deduced directly from the Arzelà-Ascoli theorem and the definition of $f^{[n]}$.

Therefore, by Lemma 2.5, lim $\sup _{n \rightarrow \infty} \mathscr{D}^{v[n]}$ contains an unbounded component $\mathscr{D}^{v} \mathrm{em}$ anating from $\left(\frac{\lambda^{v}}{r f_{0}}, 0\right)$ and joining to $(0, \infty)$.

From $\lim _{n \rightarrow+\infty} f^{[n]}(s)=f(s),(5.8)$ can be converted to the equivalent equation (5.2). Since $\mathscr{D}^{v[n]} \subset\left(\mathbb{R} \times P^{v}\right)$, we conclude $\mathscr{D}^{v} \subset\left(\mathbb{R} \times P^{v}\right)$. Moreover, $\mathscr{D}^{v} \subset \Sigma^{v}$ by (5.1).

Thus, there is an unbounded component $\mathscr{D}^{v}$ of problem (5.1) emanating from $\left(\frac{\lambda^{v}}{r f_{0}}, 0\right)$ and joining to $(0, \infty)$.

Theorem 5.4 Let (H1), (H2), (H5), and (H9) hold. For $v \in\{+,-\}$, assume that one of the following conditions holds:

(i) There exists a $\lambda_{v}^{v}>0$ for $\lambda^{v}>0$, such that $r \in\left(\lambda_{v}^{v},+\infty\right)$.

(ii) There exists a $\nu \lambda_{v}^{v}>0$ for $\nu \lambda^{v}>0$, such that $r \in\left(-\infty, \lambda_{-}^{-}\right) \cup\left(\lambda_{+}^{+},+\infty\right)$.

(iii) There exists a $v \lambda_{v}^{v}<0$ for $v \lambda^{v}<0$, such that $r \in\left(-\infty, \lambda_{+}^{+}\right) \cup\left(\lambda_{-}^{-},+\infty\right)$.

(iv) There exists a $\lambda_{v}^{v}<0$ for $\lambda^{v}<0$, such that $r \in\left(-\infty, \lambda_{v}^{v}\right)$.

Then problem (5.1) possesses two solutions $x^{+}, x^{-}$, such that $v x^{v}>0$.

Proof We shall only prove the case of (i) since the proofs of the cases for (ii), (iii), and (iv) are completely analogous.

Define

$$
f^{[n]}(s):= \begin{cases}n \varphi_{p}(s), & s \in(-\infty,-2 n] \cup[2 n,+\infty), \\ \frac{n \varphi_{p}(2 n)+f(-n)}{n}(s+n)+f(-n), & s \in(-2 n,-n), \\ \frac{n \varphi_{p}(2 n)-f(n)}{n}(s-n)+f(n), & s \in(n, 2 n), \\ f(s), & s \in\left[-n,-\frac{2}{n}\right] \cup\left[\frac{2}{n}, n\right], \\ -\left[f\left(-\frac{2}{n}\right)+\frac{1}{n^{p-2}}\right](n s+2)+f\left(-\frac{2}{n}\right), & s \in\left(-\frac{2}{n},-\frac{1}{n}\right), \\ {\left[f\left(\frac{2}{n}\right)-\frac{1}{n^{p-2}}\right](n s-2)+f\left(\frac{2}{n}\right),} & s \in\left(\frac{1}{n}, \frac{2}{n}\right), \\ n \varphi_{p}(s), & s \in\left[-\frac{1}{n}, \frac{1}{n}\right] .\end{cases}
$$

We consider the following problem:

$$
\begin{aligned}
& -\left(\varphi_{p}\left(x^{\prime}\right)\right)^{\prime}+q(t) \varphi_{p}(x)=\alpha(t) \varphi_{p}\left(x^{+}\right)+\beta(t) \varphi_{p}\left(x^{-}\right)+\lambda r a(t) f^{[n]}(x), \quad t \in(0, T), \\
& x(0)=x(T), \quad x^{\prime}(0)=x^{\prime}(T) .
\end{aligned}
$$


Clearly, we can see that $\lim _{n \rightarrow+\infty} f^{[n]}(s)=f(s),\left(f^{[n]}\right)_{0}=n$, and $\left(f^{[n]}\right)_{\infty}=n$.

Applying a similar method to the proof of Theorem 5.2, we obtain an unbounded component $\mathscr{D}^{v} \subset \Sigma^{v}$ with $(0,0) \in \mathscr{D}^{v}$.

Similar to the proof of Theorem 5.3, we can show that $(0, \infty) \in \mathscr{D}^{\nu}$.

Theorem 5.5 Let (H1), (H2), (H5), and (H10) hold. For $v \in\{+,-\}$, assume that one of the following conditions holds:

(i) $r \in(0,+\infty)$ for $\lambda^{v}>0$.

(ii) $r \in(0,+\infty) \cup(-\infty, 0)$ for $\nu \lambda^{\nu}>0$ or $\nu \lambda^{\nu}<0$.

(iii) $r \in(-\infty, 0)$ for $\lambda^{v}<0$.

Then problem (5.1) possesses two solutions $x^{+}, x^{-}$, such that $v x^{v}>0$.

Proof We shall only prove the case of (i) since the proofs of the cases for (ii), (iii), and (iv) are completely analogous.

Define

$$
f^{[n]}(s):= \begin{cases}n \varphi_{p}(s), & s \in(-\infty,-2 n] \cup[2 n,+\infty), \\ \frac{n \varphi_{p}(2 n)+f(-n)}{n}(s+n)+f(-n), & s \in(-2 n,-n), \\ \frac{n \varphi_{p}(2 n)-f(n)}{n}(s-n)+f(n), & s \in(n, 2 n), \\ f(s), & s \in\left[-n,-\frac{2}{n}\right] \cup\left[\frac{2}{n}, n\right], \\ -\left[f\left(-\frac{2}{n}\right)+\frac{1}{n^{p}}\right](n s+2)+f\left(-\frac{2}{n}\right), & s \in\left(-\frac{2}{n},-\frac{1}{n}\right), \\ {\left[f\left(\frac{2}{n}\right)-\frac{1}{n^{p}}\right](n s-2)+f\left(\frac{2}{n}\right),} & s \in\left(\frac{1}{n}, \frac{2}{n}\right), \\ \frac{1}{n} \varphi_{p}(s), & s \in\left[-\frac{1}{n}, \frac{1}{n}\right] .\end{cases}
$$

We consider the following problem:

$$
\begin{aligned}
& -\left(\varphi_{p}\left(x^{\prime}\right)\right)^{\prime}+q(t) \varphi_{p}(x)=\alpha(t) \varphi_{p}\left(x^{+}\right)+\beta(t) \varphi_{p}\left(x^{-}\right)+\lambda r a(t) f^{[n]}(x), \quad t \in(0, T), \\
& x(0)=x(T), \quad x^{\prime}(0)=x^{\prime}(T) .
\end{aligned}
$$

Clearly, we can see that $\lim _{n \rightarrow+\infty} f^{[n]}(s)=f(s),\left(f^{[n]}\right)_{0}=\frac{1}{n}$, and $\left(f^{[n]}\right)_{\infty}=n$.

Similar to the proof of Theorem 5.1, there exists an unbounded continuum $\mathscr{D}^{v[n]}$ of solutions of problem (5.9) emanating from $\left(\frac{n \lambda^{v}}{r}, 0\right)$, such that $\mathscr{D}^{v[n]} \subset\left(\left(\mathbb{R} \times P^{\nu}\right) \cup\left\{\left(\frac{n \lambda^{v}}{r}, 0\right)\right\}\right)$, and $\mathscr{D}^{v[n]}$ joins $\left(\frac{n \lambda^{v}}{r}, 0\right)$ to $\left(\frac{\lambda^{v}}{r n}, \infty\right)$.

Taking $z_{n}=\left(\frac{n \lambda^{v}}{r}, 0\right)$ and $z^{*}=(\infty, 0)$, we have $z_{n} \rightarrow z^{*}$.

So condition (i) in Lemma 2.5 is satisfied with $z^{*}=(\infty, 0)$.

Obviously

$$
r_{n}=\sup \left\{\lambda+\|x\| \mid(\lambda, x) \in \mathscr{D}^{\nu[n]}\right\}=\infty
$$

and accordingly, (ii) in Lemma 2.5 holds. (iii) in Lemma 2.5 can be deduced directly from the Arzelà-Ascoli theorem and the definition of $f^{[n]}$.

Therefore, by Lemma 2.5, lim $\sup _{n \rightarrow \infty} \mathscr{D}^{v[n]}$ contains an unbounded component $\mathscr{D}^{v}$ with $(\infty, 0) \in \mathscr{D}^{v}$.

From $\lim _{n \rightarrow+\infty} f^{[n]}(s)=f(s),(5.8)$ can be converted to the equivalent equation (5.2). Since $\mathscr{D}^{v[n]} \subset\left(\mathbb{R} \times P^{v}\right)$, we conclude $\mathscr{D}^{v} \subset\left(\mathbb{R} \times P^{v}\right)$. Moreover, $\mathscr{D}^{v} \subset \Sigma^{v}$ by (5.1).

Similar to the proof of Theorem 5.3, we can show that $(0, \infty) \in \mathscr{D}^{\nu}$. 
Remark 5.6 (see [34], p.284, L.17-23) The bifurcation argument uses the implicit function theorem and relies on the linearization. This condition may fail in this paper. So, the proofs of Theorems 3.1, 4.1, 5.1 are more complicated than the proofs of Theorems 1.1, 1.2, 1.3 in [26].

Remark 5.7 In (4) of [20], the assumption $f_{0} \in(0,+\infty)$ implies that $f$ is Fréchet differentiable at the origin, i.e., $f$ is linearizable at the origin. However, in the paper $F$ is not necessarily differentiable at the origin because of the influence of the $\operatorname{term} f$. So the bifurcation theory of [20] cannot be applied directly to obtain our results.

Remark 5.8 In [20], the nonlinearities are Fréchet differentiable or $p-1$ homogeneous linearizable at the origin or infinity. However, the nonlinear term of problem (5.1) is not necessarily $p-1$ homogeneous linearizable at the origin and infinity because of the influence of the term $\alpha \varphi_{p}\left(x^{+}\right)+\beta \varphi_{p}\left(x^{-}\right)$. So the bifurcation theory of [20] cannot be applied directly to obtain our results. Luckily, using Theorems 3.1 and 4.1, we can obtain some results of the existence of one-sign solutions, which extend the corresponding ones of [20] in some sense.

Remark 5.9 When $\alpha=\beta=0$ and $f_{0}, f_{\infty} \notin(0, \infty)$, Dai et al. [20] studied bifurcation and nodal solutions for one-dimensional $p$-Laplacian problems with non-asymptotic nonlinearity at 0 or $\infty$. Thus, Theorems 5.2-5.5 extend the corresponding results of Theorems 5.2, 5.6, 5.8, 5.9, 5.12 of [20].

Competing interests

The authors declare that they have no competing interests.

\section{Authors' contributions}

WS conceived of the study, and participated in its design and coordination and helped to draft the manuscript. TH participated in the design of the study. All authors read and approved the final manuscript.

\section{Acknowledgements}

Thanks are given to the anonymous referee for his/her valuable suggestions. The authors were supported by the NSFC (No. 11561038) and Gansu Provincial National Science Foundation of China (No. 145RJZA087).

Received: 19 March 2015 Accepted: 25 January 2016 Published online: 10 February 2016

References

1. Krasnoselskii, MA: Topological Methods in the Theory of Nonlinear Integral Equations. Macmillan, New York (1965)

2. Rabinowitz, PH: Some global results for nonlinear eigenvalue problems. J. Funct. Anal. 7, 487-513 (1971)

3. Dancer, EN: On the structure of solutions of non-linear eigenvalue problems. Indiana Univ. Math. J. 23, 1069-1076 (1974)

4. Camassa, R, Holm, D: An integrable shallow water equation with peaked solitons. Phys. Rev. Lett. 71, 1661-1664 (1993)

5. Camassa, R, Holm, D, Hyman, J: A new integrable shallow water equation. Adv. Appl. Mech. 31, 1-33 (1994)

6. Constantin, A, McKean, HP: A shallow water equation on the circle. Commun. Pure Appl. Math. 52, 949-982 (1999)

7. Constantin, A: On the spectral problem for the periodic Camassa-Holm equation. J. Math. Anal. Appl. 210, 215-230 (1997)

8. Johnson, RS: Camassa-Holm, Korteweg-de Vries and related models for water waves. J. Fluid Mech. 455, 63-82 (2002)

9. Constantin, A, Lannes, D: The hydrodynamical relevance of the Camassa-Holm and Degasperis-Procesi equations. Arch. Ration. Mech. Anal. 192, 165-186 (2009)

10. Constantin, A, Johnson, RS: Propagation of very long water waves, with vorticity, over variable depth, with applications to tsunamis. Fluid Dyn. Res. 40, 175-211 (2008)

11. Atici, FM, Guseinov, GS: On the existence of positive solutions for nonlinear differential equations with periodic boundary conditions. J. Comput. Appl. Math. 132, 341-356 (2001)

12. Zhang, Z, Wang, J: On existence and multiplicity of positive solutions to periodic boundary value problems for singular nonlinear second order differential equations. J. Math. Anal. Appl. 281, 99-107 (2003)

13. Jiang, D, Chu, J, O’Regan, D, Agarwal, RP: Multiple positive solutions to superlinear periodic boundary value problems with repulsive singular forces. J. Math. Anal. Appl. 286, 563-576 (2003)

14. Li, Y: Positive doubly periodic solutions of nonlinear telegraph equations. Nonlinear Anal. 55, $245-254$ (2003) 
15. Torres, P: Existence of one-signed periodic solutions of some second-order differential equations via a Krasnosel'skii fixed point theorem. J. Differ. Equ. 190, 643-662 (2003)

16. O'Regan, D, Wang, H: Positive periodic solutions of systems of second order ordinary differential equations. Positivity 10, 285-298 (2006)

17. Graef, JR, Kong, L, Wang, H: Existence, multiplicity, and dependence on a parameter for a periodic boundary value problem. J. Differ. Equ. 245, 1185-1197 (2008)

18. $\mathrm{Ma}, \mathrm{R}, \mathrm{Xu}, \mathrm{J}, \mathrm{Han}, \mathrm{X}$ : Global bifurcation of positive solutions of a second-order periodic boundary value problem with indefinite weight. Nonlinear Anal. 74, 3379-3385 (2011)

19. $\mathrm{Ma}, \mathrm{R}, \mathrm{Xu}, \mathrm{J}$, Han, $\mathrm{X}$ : Global structure of positive solutions for superlinear second-order periodic boundary value problems. Appl. Math. Comput. 218, 5982-5988 (2012)

20. Dai, G, Ma, R, Wang, H: Eigenvalues, bifurcation and one-sign solutions for the periodic $p$-Laplacian. Commun. Pure Appl. Anal. 12(6), 2839-2872 (2013)

21. Berestycki, H: On some nonlinear Sturm-Liouville problems. J. Differ. Equ. 26, 375-390 (1977)

22. Schmitt, K, Smith, HL: On eigenvalue problems for nondifferentiable mappings. J. Differ. Equ. 33, 294-319 (1979)

23. Ma, R, Dai, G: Global bifurcation and nodal solutions for a Sturm-Liouville problem with a nonsmooth nonlinearity. J. Funct. Anal. 265, 1443-1459 (2013)

24. Dai, G: Global bifurcation from intervals for Sturm-Liouville problems which are not linearizable. Electron. J. Qual. Theory Differ. Equ. 2013, 65 (2013)

25. Dai, G, Ma, R: Bifurcation from intervals for Sturm-Liouville problems and its applications. Electron. J. Differ. Equ. 2014, $3(2014)$

26. Dai, G, Ma, R: Unilateral global bifurcation for $p$-Laplacian with non- $p$ - 1-linearization nonlinearity. Discrete Contin. Dyn. Syst. 35(1), 99-116 (2015)

27. Dai, G, Ma, R: Global bifurcation, Berestycki's conjecture and one-sign solutions for $p$-Laplacian. Nonlinear Anal. 91, 51-59 (2013)

28. Dancer, EN: Bifurcation from simple eigenvalues and eigenvalues of geometric multiplicity one. Bull. Lond. Math. Soc 34, 533-538 (2002)

29. López-Gómez, J: Spectral Theory and Nonlinear Functional Analysis. Chapman \& Hall/CRC, Boca Raton (2001)

30. Kusano, T, Jaros, T, Yoshida, N: A Picone-type identity and Sturmian comparison and oscillation theorems for a class of half-linear partial differential equations of second order. Nonlinear Anal. 40, 381-395 (2000)

31. Lee, YH, Sim, I: Global bifurcation phenomena for singular one-dimensional $p$-Laplacian. J. Differ. Equ. 229, 229-256 (2006)

32. Whyburn, GT: Topological Analysis. Princeton University Press, Princeton (1958)

33. $\mathrm{Ma}, \mathrm{R}, \mathrm{An}, \mathrm{Y}$ : Global structure of positive solutions for nonlocal boundary value problems involving integral conditions. Nonlinear Anal. 71, 4364-4376 (2009)

34. Binding, P, Rynne, B: Half-eigenvalues of periodic Sturm-Liouville problems. J. Differ. Equ. 206, 280-305 (2004)

35. Kielhöfer, H: Bifurcation Theory: An Introduction with Applications to PDEs. Springer, New York (2004)

36. Ambrosetti, A, Calahorrano, RM, Dobarro, FR: Global branching for discontinuous problems. Comment. Math. Univ. Carol. 31, 213-222 (1990)

\section{Submit your manuscript to a SpringerOpen ${ }^{\circ}$ journal and benefit from:}

- Convenient online submission

- Rigorous peer review

- Immediate publication on acceptance

- Open access: articles freely available online

- High visibility within the field

- Retaining the copyright to your article 